\title{
TV/Series
}

1 | 2012

Les Séries télévisées américaines contemporaines :

entre la fiction, les faits, et le réel

\section{Entre ombres et lumières : les figures de policiers en eaux troubles, symboles d'une Amérique en perte de repères (The Wire, The Shield, Dexter)}

Julien Achemchame

\section{OpenEdition}

\section{Journals}

Édition électronique

URL : http://journals.openedition.org/tvseries/1521

DOI : 10.4000/tvseries.1521

ISSN : 2266-0909

Éditeur

GRIC - Groupe de recherche Identités et Cultures

Référence électronique

Julien Achemchame, «Entre ombres et lumières : les figures de policiers en eaux troubles, symboles d'une Amérique en perte de repères (The Wire, The Shield, Dexter)", TV/Series [En ligne], 1 | 2012, mis en ligne le 15 mai 2012, consulté le 19 avril 2019. URL : http://journals.openedition.org/tvseries/1521 ; DOI : 10.4000/tvseries.1521

\section{c) (1) $(9)$}

TV/Series est mis à disposition selon les termes de la licence Creative Commons Attribution - Pas d'Utilisation Commerciale - Pas de Modification 4.0 International. 


\section{Entre ombres et lumières : les figures de policiers en eaux troubles, symboles d'une Amérique en perte de repères (The Wire, The Shield, Dexter)}

Depuis l'apparition des séries télévisées dans les années 1950, notamment aux États-Unis, le policier demeure un personnage central des fictions, témoin privilégié des mutations d'une société. Nous nous intéressons aux représentations du policier dans quelques fictions télévisées contemporaines américaines en mettant d'abord en évidence l'émergence d'une nouvelle figure de policier, complexe et trouble, apparu dans les années 90 avec l'officier Andy Sipowicz dans NYPD Blue, et bien loin des séries policières pionnières américaines telles Les Incorruptibles et Dragnet, qui mettent en avant des modèles de morale. Nous voyons ensuite, à travers cette figure nouvelle, le regard dubitatif que le pays pose sur sa propre société, de plus en plus violente. A travers les exemples de séries contemporaines majeures, The Shield, The Wire et Dexter, issues de trois réseaux câblés distincts (FX, HBO et Showtime), nous analysons les différentes figures nouvelles du policier, incarnation troublée de l'ordre et de la loi.

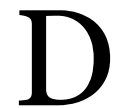

epuis leur apparition sur le petit écran américain, durant les années 1950, les séries télévisées ne cessent de faire du policier (ou du « flic ») un personnage central des fictions. Le «cop», incarnation de la loi et de ses applications morales, demeure ainsi le témoin privilégié des mutations d'une société et de ses travers les plus intimes. En effet, les "Cop Shows ", séries narrant le quotidien des forces de l'ordre, sont légions dans l'histoire de la télévision américaine et témoignent, au fil des saisons et des ans, d'une société américaine en pleine mutation: de Dragnet (1951-1958, créé et interprété par Jack Webb) à Detroit 1-8-7 (créé en 2010 par Jason Richman) en passant par Les Incorruptibles (1959-1963, créé par Quinn Martin), Columbo (1971-2003, créé par William Link et Richard Levinson), Hill Street Blues (1981-1987, créé par Steven Bochco et Michael Kozoll), Law and Order (1990-2010, créé par Dick Wolf) ou Les Experts (créé en 2000 par Anthony E. Zuiker), etc. les exemples sont extrêmement nombreux. Apparues alors que le Code (de censure) Hays était encore en vigueur, les séries policières devaient présenter aux téléspectateurs des gages de bonne moralité. En effet, le fameux « code » du sénateur William Hays, élaboré à la fin des années 1920 et mis en application au début des 
années 1930, trouvait sa légitimité dans un contexte de scandales sulfureux au sein de l'industrie cinématographique hollywoodienne : le procès pour viol et meurtre de la star du burlesque Roscoe « Fatty » Arbuckle ou encore l'assassinat du réalisateur William Desmond Taylor. Souhaitant montrer à l'image la bonne morale en action, le code Hays, dans la première section de ses recommandations particulières, s'intéressait spécialement à la représentation du crime :

These shall never be presented in such a way as to throw sympathy with the crime as against law and justice or to inspire others with a desire for imitation. The technique of murder must be presented in a way that will not inspire imitation. Brutal killings are not to be presented in detail. Revenge in modern times shall not be justified. Illegal drug traffic must never be presented ${ }^{1}$.

Les premières séries télévisées policières respectent les impératifs du Code et nous montrent des figures d'agents des forces de l'ordre dont la bonne moralité force l'admiration et le respect alors qu'ils sont confrontés aux crimes violents de la cité : il suffit de penser au modèle véridique Eliot Ness et sa bande d'Incorruptibles combattant le terrible $\mathrm{Al}$ Capone et ses acolytes patibulaires dans le Chicago de l'époque de la Prohibition, ou bien encore au détective Joe Friday, dans Dragnet, démasquant meurtriers dans le Los Angeles des années 1950. Même si le Code Hays disparaît en 1966, il faut attendre le milieu des années 1990, sur le network ABC, pour découvrir une figure de policier ambiguë et loin du modèle de l'incorruptible : il s'agit d'Andy Sipowicz, l'inspecteur du 15ème (et imaginaire) Precinct de New-York et personnage central de la série NYPD Blue, créée en 1993 par Steven Bochco et David Milch. Acclamée par la critique et l'intelligentsia, comme le fut en son temps Hill Street Blues du même Steven Bochco, notamment pour son esthétique réaliste et l'acuité de son regard sur la société américaine (on y observe l'émergence de la délinquance des gangs et une peinture réaliste de la famille américaine moderne), la série NYPD Blue propose en effet un personnage de policier extrêmement complexe. Alcoolique, irascible et au tempérament violent, Andy Sipowicz fait date dans l'histoire de la représentation du " flic » sur le petit écran et ouvre une brèche dans laquelle les chaînes de télévision américaines vont s'engouffrer. Autre facteur important permettant l'émergence que cette nouvelle "sorte de flics ${ }^{2}$, à la morale plus trouble: l'arrivée massive dans les foyers

1 On retrouvera le texte original du code Hays sur le site suivant :

http://www.artsreformation.com/aoo1/hays-code.html (consulté le 11/03/2011)

2 « Good cop and bad cop have left for the day. I'm a different kind of cop » dit l'inspecteur 
américains des chaînes câblées tels HBO, Showtime et FX, proposant des séries novatrices et non soumises à la censure contrairement aux networks historiques.

Nous voyons aujourd'hui le chemin parcouru depuis les années 1950 et nous ne pouvons que constater l'apparition d'une nouvelle figure du policier à la morale bien plus trouble dans les fictions télévisées américaines contemporaines. Nous souhaitons donc ici nous intéresser aux mutations de cette représentation du « flic » dans trois séries récentes qui, grâce à leurs qualités esthétiques et narratives, apparaissent comme des succès critiques et/ou populaires : The Wire / Sur écoute (2002-2008, créé par David Simon), The Shield (20022008, créé par Shawn Ryan) et Dexter (créé en 2006 par James Manos, Jr.), issues de trois réseaux câblés distincts (respectivement HBO, FX et Showtime). Nous analyserons ces différentes figures du policier, incarnation troublée de l'ordre et de la loi et nous les confronterons au réel dont elles peuvent être issues. Nous verrons ainsi comment elles témoignent d'une société américaine contemporaine confrontée à une violence omniprésente et qui, forte de ce constat, se retrouve en plein questionnement moral.

\section{The Wire : chronique urbaine et désenchantée sur l'Amérique}

The Wire (Sur écoute en français) apparaît sur les écrans de la chaîne HBO le 2 juin 2002 et nous narre en détails l'enquête d'un groupe d'inspecteurs chargés de surveiller et d'arrêter des dealers dans la ville de Baltimore. Proposant, sans jugement et sans concession, un aller-retour permanent entre les dealers et les policiers, la série témoigne d'une grande originalité dans son approche des situations et dans son traitement narratif. Ancien journaliste du Baltimore Sun chargé des affaires criminelles, David Simon, créateur de la série, offre à la chaîne câblée un projet ambitieux :

Je leur ai proposé de peindre en détail «la guerre de la drogue ». De "construire" une ville, secteur par secteur. De décrire la violence et le dysfonctionnement des bureaucraties qui se développent sur chaque versant de la loi, chez les trafiquants comme dans les institutions. D'analyser la culture de la dépendance - à la drogue comme au pouvoir - et les ravages d'un capitalisme qui a perdu tout visage humain ${ }^{3}$.

Vic Mackey à un suspect qu'il est chargé de "faire parler » à l'aide de ses méthodes " musclées » dans l'épisode pilote de The Shield.

3 Propos de David Simon in Laurent Rigoulet, "The Wire, la série qui peut sauver 
D'emblée nous voyons ici l'ambition politique et sociale de l'ancien journaliste, qui souhaite ainsi utiliser le medium télévisuel pour scruter le (dys)fonctionnement social de Baltimore, la ville du Maryland étant la synecdoque d'une Amérique en perte de vitesse et dont le modèle social n'apparaît plus viable. La série possède l'allure de la chronique, elle prend le temps d'analyser dans sa profondeur, de montrer précisément les secrets rouages de la ville, de ne pas céder à la tentation du cliffhanger, pourtant si caractéristique de la production télévisuelle contemporaine. $\mathrm{Au}$ contraire, le récit de The Wire fonctionne à la manière impressionniste, par successions de séquences, parfois digressives, mais qui nous font plonger dans la réalité quotidienne d'un lieu, nous en font sentir l'atmosphère. La série est ainsi co-écrite par Ed Burns, qui fut inspecteur de police au sein du département des affaires criminelles entre 1975 et 1995, avant de passer ensuite quelques années en tant qu'enseignant dans un collège du centre-ville de Baltimore. Burns était d'ailleurs la source principale de David Simon lorsqu'il travaillait au Baltimore Sun. La synergie de l'expérience des deux hommes permet à la série de se nourrir du réel comme aucune autre série. De plus, les deux compères vont passer un an à écouter les habitants des ghettos de Baltimore et ainsi puiser le matériau de leur récit immense, dont l'ébauche est la mini-série The Corner, diffusée à l'été 2000 sur HBO. Pour David Simon le constat est implacable :

[C'est] pendant les années Reagan [que] les choses ont vraiment mal tourné en Amérique et que l'économie du crack est devenue une des seules lignes d'horizon dans les ghettos. Aujourd'hui, 50 \% des noirs de Baltimore sont sans emploi et très souvent sans ressources car les aides sociales ont été supprimées les unes après les autres4.

Cette dure réalité américaine est le point de départ de la fiction de The Wire, son cadre général, et c'est à l'intérieur de celui-ci qu'évoluent l'ensemble des personnages. Sans manichéisme, la série brosse ainsi le portrait de dizaines de personnages, tant du côté des voyous que du côté des policiers, tous inspirés de la réalité.

Nombre de personnages de The Wire sont inspirés des policiers

l'Amérique », Télérama ${ }^{\circ} 3052$, décembre 2008.

4 Propos de David Simon in Laurent Rigoulet, "The Wire est une œuvre qui emprunte à la tragédie grecque », article publié le 16 novembre 2008, http://television.telerama.fr/television/david-simon-the-wire-est-une-uvre-qui-empruntea-la-tragedie-grecque,31593.php, consulté le 12/03/2011 
et des dealers que nous avons fréquentés à différentes époques de notre collaboration, raconte David Simon. Omar, dont Obama, a dit qu'il était son personnage préféré (mais qu'il ne souscrivait pas à ses actes) est inspiré, par exemple, par des figures très précises, Shorty Boyd, Cadillac and Low, Anthony [Hollie]5... Des « artistes du braquage » qu'Ed Burns connaissait bien et dont la spécialité était de voler les dealers. Dans le feuilleton, certains " gangsters ", retirés des voitures, font même des apparitions et jouent des rôles de composition ${ }^{6}$.

Larry « Donnie » Andrews, par exemple, était un braqueur de dealers connu à Baltimore dans les années 1980. Il fut condamné pour meurtre en 1986 et fut libéré en 2005.

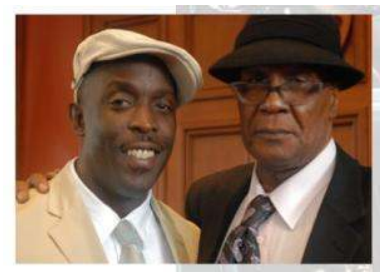

Larry « Donnie » Andrews était un braqueur de dealers connu à Baltimore dans les années 1980. Il fut condamné pour meurtre en 1986 et fut libéré

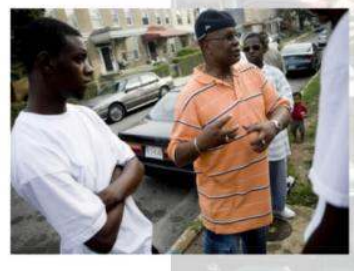
en 2005 . Il est un des modèles du personnage d'Omar Little et apparaîtra dans la série... aux côtés de ce personnage...

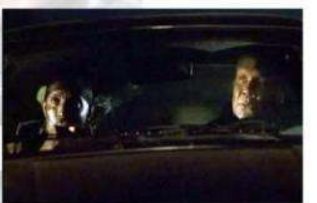

Il est aussi l'un des modèles du personnage d'Omar Little et apparaîtra dans la série en 2006, aux côtés de ce personnage dans trois épisodes de la saison 4 (épisodes 6, 7 et 12). De la même façon, «Little Melvin » Williams, baron de la drogue à Baltimore dans les années 1970 et 1980, demeure l'un des modèles d'Avon Barksdale.

5 Dans une interview au site internet du Guardian paru le 13/01/2005, David Simon indique que la figure d'Omar Little est inspiré de Shorty Boyd, Donnie Andrews, Ferdinand Harvin, Billy Outlaw and Anthony Hollie, http://blogs.guardian.co.uk/theguide/archives/tv and radio/2005/01/totally wired.html , consulté le 03/05/2011.

6 Ibid. 


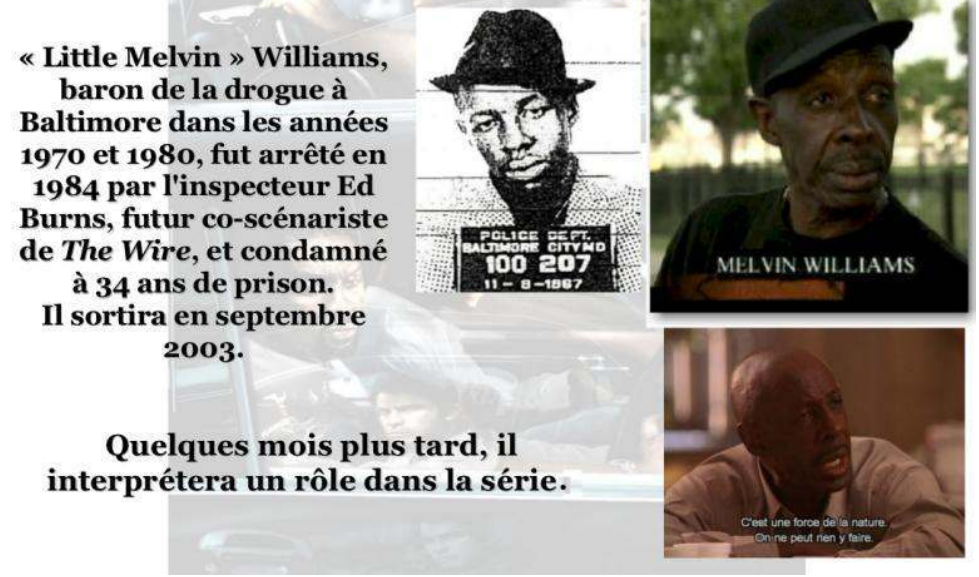

Il fut arrêté en 1984 par l'inspecteur Ed Burns et condamné à 34 ans de prison. Il sortira en septembre 2003 et interprétera un petit rôle récurrent de leader associatif dans une dizaine d'épisodes de la série (saison 3 épisodes 2, 5, 7, 8, 9, 10 ; saison 4 épisodes 3, 4, 6, 12 ; saison 5 épisode 9). De même, Edward T. Norris fut le chef de la police de Baltimore entre mars 2000 et décembre 2002 puis chef de la police de l'Etat du Maryland jusqu'en décembre 2003. A cette période il aura même quelques problèmes avec la justice ${ }^{7}$ et sera condamné en mars 2004.

7 http://www.baltimoresun.com/news/balte.md.norriso9maro9,0,5325678.story?coll=balhome-headlines, consulté le 20/06/2011. 


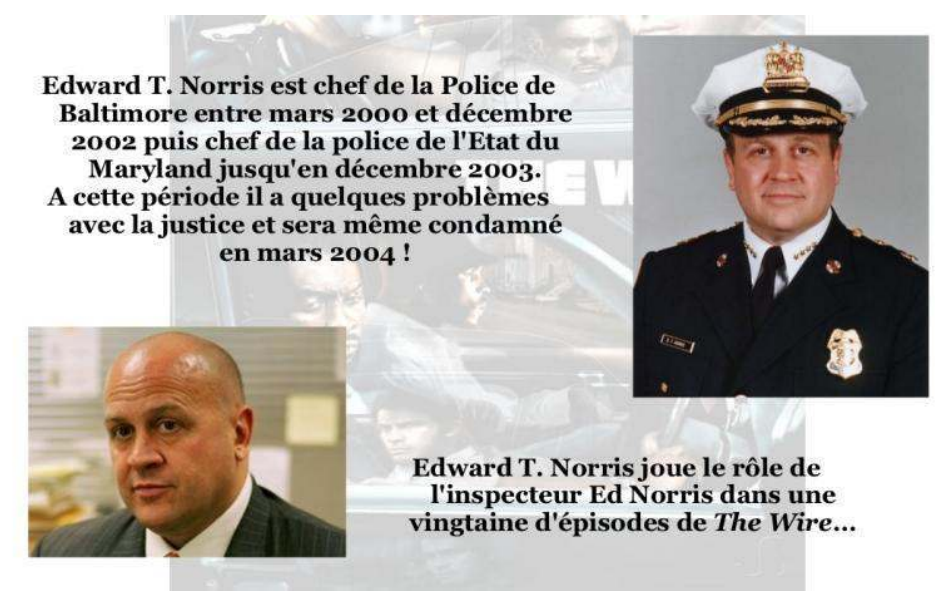

Ce dernier interprète le rôle de l'inspecteur Ed Norris dans près d'une vingtaine d'épisodes de The Wire, dès la première saison. Enfin, le révérend Frank M. Reid III et même l'ancien maire de Baltimore Kurt Schmoke, ainsi que l'ancien gouverneur du Maryland, Robert L. Ehrlich Jr. interpréteront de manière souvent très ponctuelle voire anecdotique de petits rôles ou feront même simplement de la figuration dans la série.

Le lien entre fiction et réalité est donc finalement très étroit et il témoigne d'une envie d'entrer dans la complexité psychologique des personnages, de les inscrire dans un contexte politique et social très précis, dont ils font partie et qui, surtout, les écrase. Grâce à ses acteurs non-professionnels, personnalités locales ou enfants des rues, dont le langage caractéristique et la vérité permettent de rendre les situations immédiatement crédibles, la série propose une fiction télévisuelle plus proche de l'esthétique néo-réaliste que de celle d'Hollywood. Quant à la représentation des forces de l'ordre, elle est, elle aussi, sans concession. Pas question de magnifier la police ou de la critiquer mais de la donner à voir comme elle est, dans toute sa complexité, avec ses qualités et ses défauts ou ses dysfonctionnements.

La police n'est pas héroïque. Elle travaille. Les dealers ne sont pas monstrueux. Ils travaillent. C'est peut-être la chose la plus impressionnante, cette répartition des tâches, cet incessant aller-retour entre les deux points de vue. Pur champ-contrechamp. Même valeur de cadre, à hauteur d'homme, fordien. On passe de l'un à l'autre en se 
surveillant. ${ }^{8}$

Autrement dit, il n'y a pas de méchants ni de héros, pas de mal, ni de bien, simplement des hommes (et quelques femmes) qui essayent de vivre, de s'en sortir avec ce qu'ils ont. Cette absence de jugement moral dans la structure narrative constitue la transgression la plus forte du socle des valeurs. Nous sommes face à des êtres qui luttent au sein d'un système qui les écrase sous son poids. Les deux personnages de policiers les plus intéressants de ce point de vue sont l'inspecteur de la Criminelle Jimmy McNulty (Dominic West), personnage principal de la série, et son mentor, le Major Howard "Bunny » Colvin (Robert Wisdom), responsable du Western District, quartier le plus " difficile » de Baltimore. Le premier parce qu'il est pétri de contradictions : incroyablement doué pour son travail, il est animé d'un idéal fort et ne se contente jamais du médiocre et des faibles moyens accordés à la police. Il veut toujours être efficace, atteindre les trafiquants d'envergure, au sommet de l'échelle, pas les gamins qui revendent dans la rue. Rebelle à toute forme d'autorité et porté sur la bouteille, surtout lorsqu'il se rend compte que son travail ne mène à rien (ce qui arrive souvent), Jimmy McNulty n'hésitera pas à inventer, lors de la cinquième et dernière saison de la série, un faux personnage de serialkiller de sans domicile fixe, afin de pouvoir avoir les moyens matériels de mener son enquête sur le dealer Marlo Stanfield (Jamie Hector). C'est dire si la fin justifie les moyens pour cet inspecteur singulier et à la complexité indéniable. Le deuxième personnage, le Major « Bunny » Colvin, ira peut-être encore plus loin, lors de la troisième saison, lorsqu'il instaurera, au sein de son Western Disctrict, une véritable zone de légalisation des drogues lui permettant de «nettoyer » les rues adjacentes des dealers, temporairement regroupés dans une partie du quartier totalement inhabitée. Le propos ici est vraiment sulfureux. Le Major, à six mois de la retraite, est tellement désabusé par la situation dans son quartier, par l'ampleur qu'a pris la violence et le trafic de drogue, qu'il opte pour une option radicale et très discutable. Il faut voir ici le cheminement psychologique du personnage, lors des premiers épisodes de la saison : ses allers-retours entre le COMSTAT, durant lesquels les Majors des différents quartiers de Baltimore doivent présenter leurs statistiques sur la criminalité et la délinquance au Chef de la police et à son adjoint, et durant lesquels ils sont systématiquement humiliés voire débarqués de leur poste (surtout en période électorale), et les rues de son District où les corners kids lui

8 Bertrand Bonello, "The Wire (Sur écoute). Il était une fois en Amérique », Cahiers du cinéma, ${ }^{\circ} 648$, décembre 2009, p. 72-73. 
proposent une dose de crack alors qu'il arbore son uniforme de policier. Pour lui, la lutte contre la drogue est tout simplement impossible et il va faire le compromis le plus violent qui soit pour un représentant des forces de l'ordre, une sorte d'anti-prohibition, en légalisant, sur une zone précise, la vente et l'usage de drogues. Cette décision radicale, il la justifie auprès des officiers dont il a le commandement grâce à une anecdote : celle des sacs en papiers dans lesquels on dissimule les bouteilles d'alcool et qui permettent, malgré la loi, que les gens consomment de l'alcool dans la rue.

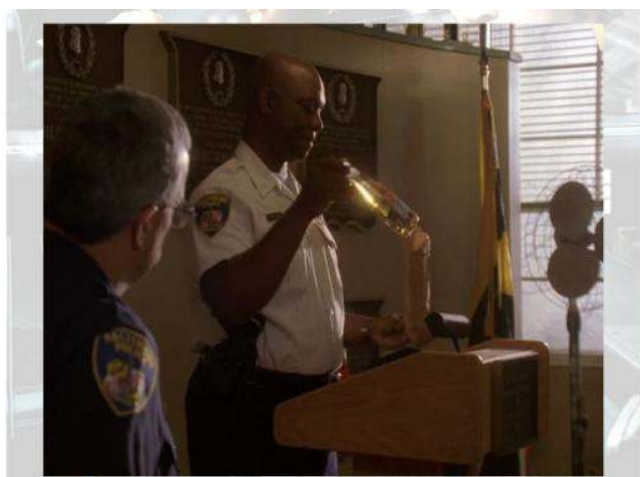

L'anecdote du sac en papier

Le « compromis civil » et la « légalisation» de la drogue

C'est ainsi que va se mettre en place, sous les yeux ébahis des spectateurs, cette zone de non-droit que les dealers s'approprient sous le nom de Hamsterdam, en référence à Amsterdam, et à sa politique de tolérance à l'usage du cannabis. Les policiers du Western District, bien évidemment réticents à cette " expérimentation », vont être chargés de faire migrer les dealers de leurs corners vers Hamsterdam, et offrent à la série des scènes souvent cocasses. Cet exemple radical pour traiter le problème de la drogue, avec quelques succès et ses échecs, sera bien évidemment sévèrement réprimé par la hiérarchie lorsqu'elle en aura connaissance et le Major sera rétrogradé et mis à la retraite de manière anticipée, afin de sauvegarder les apparences dans cet univers de dupes. Mais quelque chose de nouveau aura été tenté face à une situation intolérable. On voit bien ici qu'il ne s'agit plus, pour la police, de combattre la drogue mais d'en réduire le plus possible les effets sur les riverains. Sombre constat sur l'Amérique. 


\section{The Shield : la corruption policière et le clivage du moi}

The Shield, lorsqu'elle apparaît sur les écrans de la chaîne FX en mars 2002, montre une nouvelle incarnation du policier comme personnage principal d'une série télévisée : celle du flic corrompu. A cinquante années d'intervalle, il est intéressant de noter le basculement violent opéré entre le générique de Dragnet et celui de The Shield, deux séries dont l'action prend place à Los Angeles : un même insigne (le « shield ») revient, incarnation symbolique de l'ordre et de la loi, mais propose plutôt désormais l'éclatement, la noirceur et le chaos d'une institution américaine dont l'autorité apparaît en perte de repères.
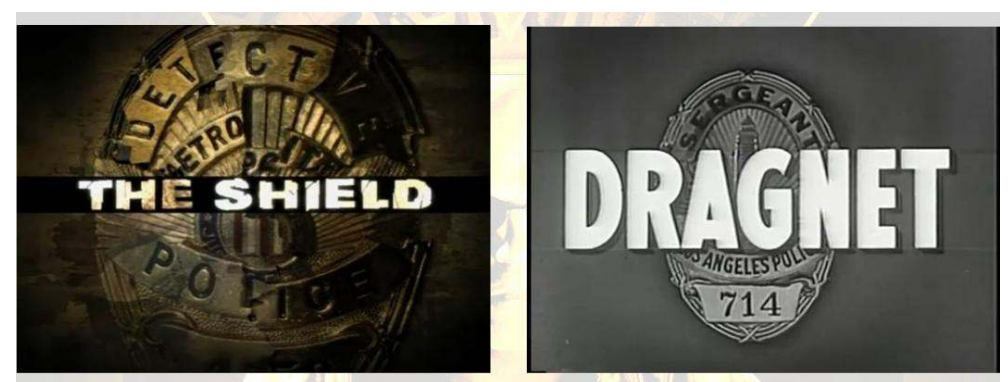

De Dragnet (1951-1958) à The Shield (2002-2008) L'insigne-symbole de l'autorité : noirceur et chaos d'une Amérique en décomposition

Personnage d'une complexité extraordinaire, l'inspecteur Vic Mackey (Michael Chiklis) est la figure trouble et centrale de la série créée par Shawn Ryan. Dès l'épisode pilote, qui se clôt par le meurtre d'un policier par Vic Mackey, toute l'ambiguïté morale est mise en lumière. Nous sommes face à un retournement violent de la morale, de la loi et de la justice. Nous découvrons un flic tueur de flic et toute la série sera hantée par ce meurtre originel montrant l'aporie d'un système policier qui secrète en son sein son propre mal. Pourtant Mackey n'est pas un personnage simpliste, il ne représente pas le mal incarné, une figure monstrueuse (et donc, de ce point de vue, 
rassurante pour le spectateur). Flic à l'éthique ambiguë, il est insaisissable : préférant la légitimité à la légalité 9 , il est un ardent protecteur de la veuve et de l'orphelin, tout en étant ultraviolent avec les délinquants avec lesquels il peut néanmoins parfois collaborer. Mackey est aussi, et surtout, intéressé par sa propre personne (il est attiré par l'appât du gain, fait payer sa protection à certains dealers et volera avec ses coéquipiers l'argent sale de trafiquants arméniens). Vic Mackey apparaît comme le symptôme possible d'une société contemporaine, privilégiant l'individualisme à outrance (Vic Mackey finira seul). Néanmoins Mackey n'est pas un sociopathe ou un psychopathe, qui fuirait toute compagnie humaine ou rapport social, il est un chef de meute, sa Strike Team (ou Brigade de choc, en français), un père de famille et un mari, et surtout, un excellent policier qui a réussi à faire baisser la criminalité dans le quartier dont il a la charge. Mais tout ceci à quel prix ? L'inspecteur Mackey laisse plutôt apparaître le mécanisme de défense que l'on nomme en psychanalyse le « clivage du $\mathrm{moi}^{10}$ » : il ne fait plus la différence entre la satisfaction de sa pulsion (appât du gain, réussite professionnelle...) et l'exigence du réel (respect de la loi, être un bon père de famille...), il ne distingue plus ce qui est bien de ce qui est mal (tout cela est terriblement relatif et dépend des circonstances comme des buts à atteindre) alors qu'il est lui-même supposé être le garant de la distinction entre les deux. Il ne met pas en lien ses propres actes illégaux avec la morale et la loi qu'il doit faire respecter, ce sont deux choses qu'il arrive à maintenir éloignées. Et cela est d'autant plus ambigu que certaines de ses activités illégales permettent de rendre une certaine forme de justice. Le personnage du flic corrompu fonctionne ici comme parangon d'une société en perte de repères stables. A l'inverse du hors-la-loi avec de grands principes (tel Omar Little dans The Wire), il est un gardien de la loi, sans principes moraux globaux : il ne vit que dans l'urgence des situations, dans un monde violent sans vérité absolue, si ce n'est celle de sa propre survie.

La force de la série, filmée d'une caméra à l'épaule tremblante, réside dans l'ambiguïté de son personnage central autant que dans ces récits shakespeariens à la noirceur tragique. Cette force est accentuée par le fait que la série a trouvé sa genèse dans le réel et fameux scandale de la Division Rampart au début des années 2000 au sein du

9 Séverine Barthes, «The Shield » in Martin Winckler (dir.), Les Miroirs obscurs, Au Diable Vauvert, Paris, 2005, p. 274.

10 Cf. Sigmund Freud, "La Scission du moi dans le processus de défense », 1938, traduit par Thierry Simonelli, http://www.psychanalyse.lu/articles/FreudScission.htm, consultée le $12 / 03 / 2011$ 
LAPD (Los Angeles Police Department). En effet, The Shield était originellement appelé Rampart avant de choisir, sous la pression des policiers ne souhaitant pas voir leur image salie plus qu'elle ne l'était déjà, une approche plus fictionnelle en situant l'action de la série dans le quartier fictif de Farmington. Néanmoins les références au scandale Rampart sont nombreuses dans la série. Cette affaire de corruption, une des plus importantes dans l'histoire de la police de Los Angeles, prend place dans le commissariat de Rampart, situé au Nord-Ouest du centre-ville et couvrant les quartiers d'Echo Park et de Westlake. Au sein de ce poste de police, une unité antigang connue sous l'acronyme CRASH (Community Ressources Against Street Hoodlums), à l'image de la Strike Team, va se distinguer par ses pratiques illégales et criminelles. Ainsi, en septembre 1999, un officier du CRASH, Rafael Perez, est arrêté par le FBI, pour avoir volé de la cocaïne dans les locaux de la police et pour l'avoir revendue. Suite à cette interpellation, l'officier Perez dévoile l'ensemble des activités illégales de son unité d'intervention : au total ce seront près d'une centaine de policiers et d'inspecteurs qui seront impliqués par le témoignage. On découvre alors que les policiers se livraient à de faux témoignages couvrant des bavures, agressions, braquage de banque, trafic de drogues et même meurtres. Tous ces éléments se retrouvant peu ou prou dans The Shield. Ainsi, le 12 octobre 1996, Rafael Perez accompagné de son équipier Nino Durden, procède à l'arrestation de Javier Ovando, 19 ans et membre d'un gang. Face à l'attitude peut-être un peu récalcitrante du jeune homme, l'officier Durden s'énerve et lui tire une balle dans la tête. Maquillant leur «bavure » en riposte de légitime défense, tout cela en plaçant une arme à côté du corps, les policiers vont réussir à faire condamner Javier Ovando, paralysé depuis, à 23 ans de prison ${ }^{11}$. Cet aspect de l'affaire se retrouve dans la trame narrative de l'épisode 9 de la saison 1 intitulé «Erreur sur la cible» (Throwaway) durant lequel Curtis Lemansky (Kenneth Johnson), un membre de la Strike Team, va tirer par accident sur un jeune homme désarmé qu'il croyait affilié à un gang. Vic Mackey, pour protéger son équipier, utilisera la même méthode que Rafael Perez en plaçant une arme dans la main du jeune homme. Il en est de même pour le trafic de drogues. Dans l'épisode 5 de la première saison intitulé « Deux Kilos » (Blowback), la Strike Team, après avoir placé une écoute illégale dans un repaire de trafiquants arméniens, va subtiliser deux kilos de cocaïne lors d'une descente de la police et tout cela sous les yeux d'une jeune recrue, l'officier Julian Lowe (Michael Jace), qui va ensuite devenir l'objet d'un chantage. De même, l'officier Rafael Perez est soupçonné d'avoir

11 http://www.laweekly.com/1999-10-21/news/naming-names/ (consulté le 24/10/2011) 
participé au meurtre du rappeur Notorious BIG sur ordre du producteur du label Death Row Records, Suge Knight. Et dans The Shield, Vic Mackey et son équipe collaborent avec le rappeur Kern Little (Kirk Jones aussi connu sous le nom d'artiste Sticky Fingaz) qui est aussi le fondateur du gang des One-Niners. The Shield puise donc dans l'histoire contemporaine de la police de Los Angeles pour nourrir ses récits et proposer à l'écran une figure trouble du policier moderne. A travers le personnage de Vic Mackey et grâce à la complexité des situations dans lesquelles il est pris (pression de la hiérarchie, il doit s'occuper de son fils autiste...), mais aussi grâce au temps étiré de la diffusion télévisuelle, le spectateur fait l'expérience d'une identification singulière avec une figure paradoxale de l'autorité. Ce trajet émotionnel du spectateur s'appuie sur la fascination pour l'ambivalence profonde du personnage : liant sa toute-puissance pulsionnelle et transgressive à une légitimité souvent compréhensible des ses faits et gestes.

\section{Dexter : L'expert scientifique en psychopathe}

Contrairement aux personnages que nous venons de voir, Dexter Morgan, le héros de la série Dexter apparue sur Showtime en 2006, ne semble pas, au premier abord, prendre ses racines dans la réalité mais dans l'imagination de l'écrivain américain Jeff Lindsay. Il faut par ailleurs noter que Dexter n'est pas véritablement un policier (il le dit lui-même au début de l'épisode pilote), il est un analyste scientifique au service de la police de Miami. Mais il représente indéniablement une figure de l'autorité. Toutefois, si l'on en croit le spécialiste des tueurs en série Stéphane Bourgoin ${ }^{12}$, le personnage de Dexter aurait un modèle issu du réel : un flic tueur en série de dealers nommé Manuel Pardo Jr. Ce dernier, policier modèle et père de famille, assassina neuf trafiquants de drogue à Miami à la fin des années quatre-vingts. Après son arrestation en 1988, il fut condamné à mort. Il attend actuellement son exécution dans le couloir de la mort. Il est intéressant de noter que Manuel Pardo prenait des polaroïds des meurtres qu'il commettait afin d'en prolonger le plaisir morbide, et qu'il s'est qualifié lui-même de justicier lors de son procès. Ces troublantes similitudes avec Dexter Morgan, les gouttes de sang prélevées remplaçant les photos, laissent penser que Jeff Lindsay, originaire de Miami, a su puiser dans les faits divers locaux pour nourrir son imagination et livrer ainsi un personnage en prise avec la société américaine contemporaine.

12 http://tvmag.lefigaro.fr/programme-tv/article/serie/50637/dexter-vu-par-un-experten-tueurs-en-serie.html (consulté le 24/10/2011) 
La « créature » de Lindsay, Dexter Morgan a la particularité d'être un tueur en série capable de " domestiquer » son besoin de tuer et de le diriger seulement vers les tueurs qui ont échappé au système judiciaire. Il a été en effet élevé par un père policier qui lui a inculqué un véritable " code » de conduite (le " Harry's Code ${ }^{13}$ ») qu'il applique scrupuleusement depuis lors. Dexter ne tue ainsi que des assassins, il ne tue pas d'innocents et mène d'abord une enquête minutieuse et approfondie sur sa future victime. Il doit trouver des preuves de culpabilité avant de passer à l'acte. Fin analyste scientifique, fasciné par le sang à cause d'un traumatisme infantile d'une rare violence (le meurtre de sa mère sous ses yeux), Dexter est un personnage à part : il est le premier tueur en série, héros ambigu, d'une fiction télévisée contemporaine. A travers une omniprésente voix-off et troublante d'honnêteté (possible résurgence du film noir), le spectateur peut suivre les méandres de la pensée du personnage, psychopathe sans émotion, portant son regard forcément cynique sur les mœurs humaines qu'il ne comprend pas. Simulant toutes ses émotions pour s'intégrer à la société, Dexter est parfois maladroit, attachant comme un enfant perdu dans un monde d'adulte (il apporte exemplairement des « donuts » aux policiers).

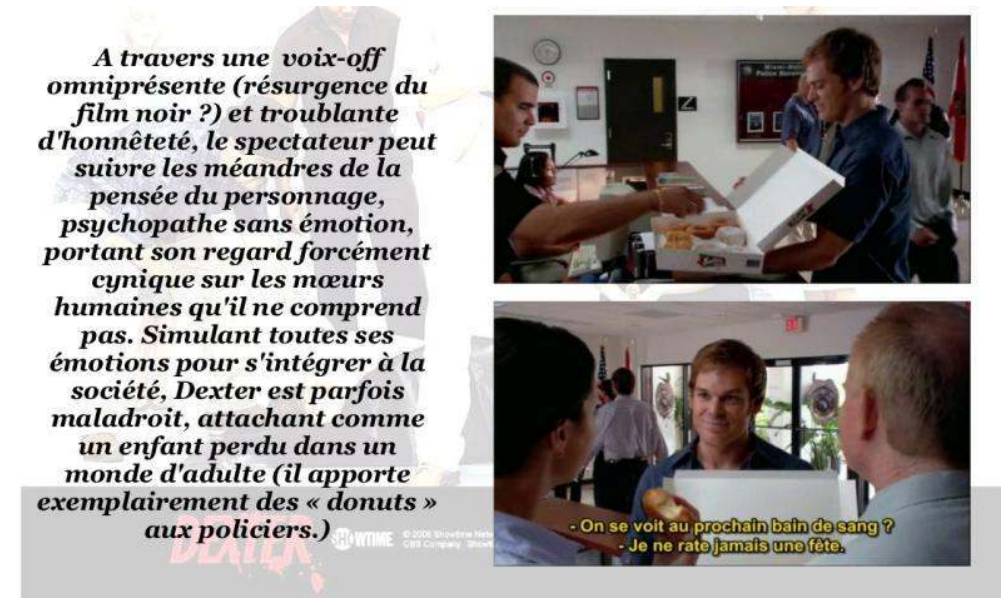

Si le personnage provoque l'empathie c'est principalement parce qu'il

13 Faut-il y voir ici une référence paronymique et ironique au « Hays Code » ? 
est fortement ambivalent : il ne peut résister à la pulsion de meurtre tapie au fond de son être mais focalise cette pulsion vers des meurtriers très dangereux et en liberté, qu'il tue d'ailleurs selon un rituel très codifié et très propre à l'écran (absence de tortures sadiques, présence de plastique pour empêcher les traces de sang). Autrement dit, Dexter Morgan est un justicier, un bourreau qui aime et prend du plaisir dans son travail. Mais le travail n'est qu'un prétexte : cela, Dexter, et le spectateur, le savent. Le personnage est fascinant par cette transgression de la morale et de la loi : il n'a point de "surmoi » et succombe à ses pulsions de destruction. Paradoxalement cette transgression de la loi et de la morale, par le meurtre, est au service d'une certaine idée de la justice. Après tout, certains états américains rendent la justice en tuant des assassins : n'est-ce pas la même pulsion meurtrière mais rationalisée, institutionnalisée ? Toute l'ambiguïté du personnage réside dans ce paradoxe et offre à la société américaine un miroir troublant tout en confrontant aussi le spectateur à ses propres pulsions individuelles. Clyde Phillips, l'un des producteurs de la série, explique :

Dexter tue, non pas pour faire régner la justice mais parce qu'il en ressent le besoin. Il ne peut pas s'en empêcher. Il est vraiment fou et très éloigné des justiciers du cinéma, comme Charles Bronson dans Un justicier dans la ville. Dexter est beaucoup plus profond, beaucoup plus complexe. Il recherche désespérément à fonder une famille car son enfance a commencé dans un bain de sang, celui de sa mère, assassinée sous ses yeux. Il se demande s'il sera un jour capable d'aimer. Puis, il se marie et devient responsable de trois enfants. Il se retrouve dans la pire position possible pour un serial killer ${ }^{14}$.

Cette quête de normalité du personnage favorise l'empathie et au fur et à mesure des saisons, le spectateur assiste fasciné à une tentative d'humanisation du psychopathe. Psychopathe paradoxal, Dexter occupe une fonction finalement fortement morale et donc terriblement ambiguë : figure toute-puissante fascinante, il exécute les assassins car son père policier a domestiqué ainsi sa pulsion meurtrière. Il nous renvoie à notre propre humanité, luttant sans cesse avec la morale patriarcale transmise par la société. En résumé, Dexter est un monstre attachant car il tue des monstres encore pire que lui. Il apparaît comme le sombre constat d'une humanité contemporaine désormais sans refoulement, prise dans les rêts de pulsions toujours

14 Propos de Clyde Phillips in Alain Carrazé, «Pourquoi Dexter n'est pas une série racoleuse? ", 03/03/2011, http://fr.tv.yahoo.com/blog/series/article/2871/pourquoidexter-nest-pas-une-srie-racoleuse.html, consulté le 05/03/2011. 
plus faciles à assouvir, mais qui doit tout de même continuer à vivre ensemble, en apparences.

Ces trois séries récentes, tout en renouvelant les codes narratifs et esthétiques de la série policière, puisent à des degrés divers leur matière dans le réel. Elles proposent, chacune à leur façon, une représentation troublée de la figure policière qui fait écho aux questionnements de la société américaine sur la violence qu'elle sécrète, et cela, à travers la représentation de l'institution principale chargée de la réguler. Grâce à ce personnage du policier à la morale ambiguë, les fictions télévisuelles contemporaines font preuve de maturité et démontrent qu'elles affrontent de manière directe la société, tendant ainsi un miroir ténébreux aux spectateurs. 